\title{
METODOLOGÍAS INNOVADORAS PARA LA ENSEÑANZA EN GESTIÓN DE SERVICIOS DE SALUD
}

\section{Maritza Martínez, MPH/ MD}

En agosto del año 2002, la rectoría del INTEC autorizó la creación del Programa de Salud y Seguridad Social (PROISS); este nace como un espacio del Área de Ciencias de la Salud para el apoyo y el fortalecimiento del Sistema Nacional de Salud (SNS) y para la implementación, desarrollo, monitoreo y medición del impacto del Sistema Dominicano de Seguridad Social (SDSS).

La misión del PROISS es ofrecer servicios de formación, capacitación e investigación al personal de Salud, en el marco regulatorio del Sistema Nacional de Salud y del Sistema Dominicano de Seguridad Social mediante la formulación de propuestas innovadoras. También pretende alcanzar la sistematización de los procesos, la auditoría social y elaboración de materiales educativos.

El espacio creado está formado por un equipo multidisciplinario, que cuenta con años de experiencia en medicina, ciencias ambientales, humanidades, economía, derecho, sociología, psicología y trabajo social. En fin, por profesionales conscientes del desafío que implica el proceso de Reforma del Sector Salud y la implementación de la Seguridad Social en Salud, la responsabilidad social y el compromiso con la acción intersectorial y la participación social en Salud. 
Creemos que el trabajo mancomunado hará posible construir un mundo mejor. Por eso estamos abiertos a las alianzas y al trabajo conjunto con personas e instituciones.

En mayo del año 2003 iniciamos una especialidad en Gestión de Servicios de Salud y Seguridad Social, para entonces se asumió el compromiso de brindar un espacio en la universidad orientado hacia la formación de los recursos humanos de acuerdo a las necesidades del país; a las competencias técnicas, las actitudes y los valores. Todo ello se hizo con la finalidad de responder a la demanda del servicio y contribuir a la resolución de las necesidades de la población y de la universidad INTEC, la cual es el resultado de las inquietudes comunes de un grupo de profesores universitarios dominicanos que, en 1971, tomaron la decisión de fundar "una pequeña institución orientada principalmente al ofrecimiento de programas de postgrado en áreas no tradicionales".

Este postgrado nace en una Universidad innovadora y que propugna por la excelencia académica; que es pionera en la oferta curricular de postgrados y cursos de Educación Permanente.

\section{Vinculación docencia servicio: E1 caso de la formación en gerencia de servicios de salud y Seguridad Social}

Esta especialidad se orienta a los profesionales que quieran incursionar en el proceso de diseño, implementación, desarrollo y evaluación de los servicios de salud y la Protección Social de la población dominicana. La metodología educativa seleccionada para este programa implica que los participantes se involucren en "Aprender a aprender y aprender a hacer". Este modelo de aprendizaje basado en problemas de la realidad de los servicios de salud, permite que el aprendizaje sea significativo y pueda aplicarse además en la transformación de la realidad.

Hay que decir que se realizaron doce rondas de la especialidad, para un total de egresados de 309 profesionales. 
La especialización se ejecuta en cinco trimestres; está facilitada por 26 docentes, cuya virtud principal es su conocimiento del sistema de salud y del sistema dominicano de la Seguridad Social, de los cambios que se están operando en ese sector, con habilidades de comunicación y manejo del proceso de enseñanza-aprendizaje.

Durante el primer trimestre se desarrolló una actividad con el fin de crear un espacio de reflexión e intercambio, sobre los retos y desafíos vinculados a la formulación de políticas públicas en salud y a las reformas de la Seguridad Social. Como ejemplo de las actividades conexas se crea un espacio en que los alumnos y las alumnas pudieron intercambiar con los expertos invitados, sobre los retos y avances de la Superintendencia de Salud y Riesgos Laborares (SISALRIL). La metodología del evento consistió en tres presentaciones de expertos, después de las cuales los alumnos presentaron sus apreciaciones y formularon sus preguntas.

Esta especialidad se imparte con una modalidad semi-presencial; durante los viernes y sábados se realizan las sesiones presenciales, permaneciendo el participante en su ámbito laboral durante el resto de la semana, realizando el Trabajo de Campo Supervisado (TCS), en el cual se lleva a cabo un proyecto de intervención, así como la investigación-acción y el acompañamiento por tutoría individual y grupal de la supervisora o el supervisor de campo.

Esta experiencia de TCS realizada en el trabajo, incorpora en los servicios de salud la modalidad de Educación Permanente, al mismo tiempo que esta modalidad vincula a otros miembros del equipo de salud que no participan en la especialidad. De esta manera se posibilita la integración de la docencia, servicio e investigación, de modo tal que dichos servicios sean transformados según el aprendizaje y los compromisos desarrollados en los participantes.

En los trabajos de campo supervisados, los tutores propician la reflexión sobre los conceptos revisados en las sesiones presenciales, clarifican dudas o amplían la información sobre los temas 
o documentos de consulta entregados. Además brindan apoyo y acompañan durante todo el proceso de detección de la problemática que se analiza, así como lo relacionado con la selección de la metodología de investigación adecuada a cada caso, su aplicación y el análisis e interpretación necesarios para extraer conclusiones.

En cada trimestre, se hizo una revisión y construcción colectiva sobre el desarrollo de la Especialidad en Gestión de Salud y Seguridad Social, con la participación del equipo de conducción y docentes del Programa INTEC de Salud y Seguridad Social, con delegados de los participantes y representantes de becados. En estas reuniones se medía el desempeño de los estudiantes, se abordaban los contenidos estipulados de los programas de clases y las técnicas vinculadas al proceso de enseñanza aprendizaje. Todo se hizo con el fin de la continua mejora del programa académico y con el objetivo de alcanzar mejores resultados en la gestión de políticas, los programas y servicios de salud.

Todo lo anterior conforma una propuesta académica innovadora con el objetivo de desarrollar conocimientos y competencias gerenciales coherentes con la problemática de su entorno y orientada al personal de salud con responsabilidades gerenciales en el Sistema Nacional de Salud y en el Sistema Dominicano de Seguridad Social.

A continuación se presenta un esquema con los ciclos y partes del diseño curricular. 


\section{Diseño curricular constructivista}

\begin{tabular}{|c|c|c|c|}
\hline Ciclo I & \multicolumn{2}{|c|}{ Ciclo II } & Ciclo III \\
\hline $\begin{array}{l}\text { Sistemas Informáticos } \\
\text { Estadística Básica } \\
\text { Comunicación Verbal y } \\
\text { Escrita } \\
\text { Metodología de la } \\
\text { Investigación }\end{array}$ & \multicolumn{2}{|c|}{$\begin{array}{l}\text { Seguridad Social } \\
\text { Economía de la Salud } \\
\text { Teoría y Conducta } \\
\text { Organizacional } \\
\text { Epidemiología Básica } \\
\text { Legislación en Salud }\end{array}$} & $\begin{array}{l}\text { Gestión Estratégica } \\
\text { Marketing Sanitario } \\
\text { Gestión de Calidad } \\
\text { Sistemas de Información }\end{array}$ \\
\hline \multicolumn{2}{|l|}{ Ciclo IV } & \multicolumn{2}{|l|}{ Ciclo V } \\
\hline \multicolumn{2}{|c|}{$\begin{array}{l}\text { Gestión del Talento Humano } \\
\text { Gestión Financiera y Costeo } \\
\text { Gestión de Servicios Asistenciales } \\
\text { Gestión de Tecnologías }\end{array}$} & \multicolumn{2}{|c|}{$\begin{array}{l}\text { Seminario de Trabajo de investigación } \\
\text { Presentación Trabajo final }\end{array}$} \\
\hline \multicolumn{4}{|c|}{$\begin{array}{c}\text { TRABAJOS DE CAMPO SUPERVISADOS } \\
\text { Y ACTIVIDADES CONEXAS }\end{array}$} \\
\hline
\end{tabular}

\section{Proceso de enseñanza aprendizaje:}

- En la construcción del conocimiento.

- Centrado en la realidad para transformarla.

- Desarrollo del Trabajo de Campo Supervisado (TCS), como eje integrador durante toda la especialidad.

- Desarrollo de proyectos de intervención viables.

- Desarrollo de capacidades para negociación y escalar los proyectos de intervención desarrollados.

- Estrategias para crear pertenencia de todos sus actores y relacionados. 


\section{Metodologías aplicadas}

- Método Estudio-Trabajo.

- Integración Docencia, Servicio e Investigación.

- Ver, juzgar y actuar.

- Aprender-haciendo.

- Aprendizaje basado en problemas.

- Diseño del proceso enseñanza-aprendizaje andragógico.

- Trabajos de Campo Supervisado (TCS).

- Actividades conexas: Encuentro con los diferentes actores del Sistema Nacional de Salud y del Sistema Dominicano de Seguridad Social al final de cada trimestre. Para el desarrollo de la especialidad, se trabajó en un proceso que inició con la consolidación del equipo docente. Lo que conllevó realizar:

$>$ Homologación de los contenidos, metodologías, técnicas didácticas de los docentes e investigadores del programa de la especialidad, impartido por el Instituto de Ciencias de la Salud (CES), de Medellín, Colombia; con el apoyo financiero del Proyecto REDSALUD.

$>$ Espacio de reflexión sobre las Reformas de Salud, Sistemas de Seguridad Social y el impacto en la formación de Recursos Humanos.

> Diplomado de Gestión de la Docencia para docentes.

$>$ Vinculación de la docencia con los espacios de investigación e intervención.

$>$ Presentación y observación de los Proyectos Demostrativos de las Regiones de Salud.

Conocimiento integral del programa. 
Identificación con los objetivos y contenidos del programa.

Desarrollo del sentimiento de pertenencia al programa, al PROISS y a la Universidad.

> Conocimiento e implementación de metodologías para educación de adultos en República Dominicana.

> Aplicación de las herramientas de TCS.

\section{Trabajos de Campo Supervisado (TCS)}

El Trabajo de Campo Supervisado (TCS) tiene como objetivo el apoyo de la aplicación práctica de las metodologías, técnicas y herramientas auxiliares, facilitadas según los contenidos desarrollados en las asignaturas del postgrado en Gestión de Servicios de Salud y Seguridad Social. Se hizo de modo tal que transformara y mejorara las competencias de los participantes y en su quehacer diario, pues estos comparten con otro personal administrativo y técnico relacionado con los servicios de salud, las direcciones provinciales de Salud, las direcciones de Áreas y ARS, entre otras. Esto dio lugar a la creación de una masa crítica que impactó los procesos de reformas del Sistema Nacional de Salud y del Sistema Dominicano de Seguridad Social.

\section{Programas de intervención elaborados por los partici- pantes de la Especialidad en Gestión de Servicios de Salud y Seguridad Social}

Como parte de los TCS, los participantes de esta especialidad elaboraron un Programa de Investigación y Proyecto de Intervención; se hizo como una respuesta a los hallazgos de sus estudios y atendiendo a la problemática detectada a través de la propuesta diseñada con este fin. A continuación se presentan algunos resultados. 


\section{Resultados}

La contribución de las acciones encaminadas a lograr un mejoramiento continuo de la gestión de los establecimientos de salud está centrada en la formación de recursos humanos. La premisa que sirve de punto de partida es la necesidad de invertir en la modificación de actitudes y prácticas y en el desarrollo de capacidades, a fin de asumir la calidad como parte inherente de la cultura organizacional y del trabajo día a día.

Los egresados de la especialidad desarrollan la capacidad de trabajo en equipo y la ejecución del proceso de autoevaluación, mediante la aplicación del Marco Común de Evaluación -CAF- y el Mejoramiento Participativo de la Calidad Basada en la Evidencia en los Servicios de Salud -EPQI-.

El abordaje de la solución a los problemas encontrados en la Autoevaluación se realizó mediante el método "Mejoramiento Participativo de la Calidad Basada en Evidencias" (EPQI), el cual se apoya en el "KAIZEN", concepto de calidad originalmente desarrollado en la Industria del Japón, que se enfoca en las necesidades de los usuarios y en el mejoramiento de los procesos orientados a la obtención de resultados. Esta herramienta aborda los pasos del mejoramiento de la calidad (QI Steps) y el ciclo de planear- hacer estudiar y actuar (PDSA Cycle).

Para la priorización se utilizó el Método KJ o Diagrama de Afinidad; el análisis de las áreas de mejora se realizó mediante el Árbol de Problemas, el análisis de las causas de las áreas de mejora mediante el Diagrama de Espina de Pescado y las propuestas de mejora se trabajaron con la herramienta Árbol de Soluciones.

Los planes de Mejora e Inversión se elaboraron a partir de los resultados del autodiagnóstico, la priorización y el análisis de los problemas encontrados. Además de los resultados de la aplicación de las guías de habilitación, mediante las cuales se 
levantó la información acerca de los requerimientos mínimos en equipamiento, condiciones físicas, cantidad y capacidad de recursos humanos para la entrega de los servicios, según capacidad resolutiva.

Todo esto tuvo como resultado el diseño de planes de mejora y de inversión en los servicios de salud, y un espíritu de motivación y participación de los integrantes de cada centro de salud de donde procedían.

Las áreas de mejoras identificadas por los participantes de la especialidad fueron:

- El fortalecimiento de la gestión por procesos de los hospitales de la región.

- La elaboración de un plan para gestionar la contratación y capacitación de los Recursos Humanos de los servicios de salud.

- Disponer de un sistema de evaluación de la satisfacción de los usuarios internos y externos [con relación] a los servicios de salud.

- Implementar un sistema para gestionar la organización de los servicios de salud.

- Involucrar al personal de salud en la toma de decisiones de los servicios de salud.

- Establecer un plan de gestión de la infraestructuraremodelación.

- Adecuación del espacio físico y equipamiento de los servicios de salud. 


\section{Otras intervenciones en gestión clínica fueron:}

- Una intervención del control prenatal implementado en las dos instituciones basadas en la evidencia de que el protocolo de normas obstétricas solo se cumplía en un 50\%; se vislumbró además la necesidad de utilizar dichos protocolos como medio para la identificación temprana de un conjunto de beneficios psicológicos, biológicos y de corte social con tal de evitar morbilidad y mortalidad materna y perinatal.

- Ante la evidencia de que solo el 10\% de las madres amamantan a sus hijos y que muchos proveedores no orientan a las futuras madres sobre la importancia de la lactancia materna.

- Se diseñó una estrategia de comunicación y educación sobre la lactancia materna y la orientación sobre las respuestas a las ITS/VIH/SIDA.

- Se elaboró una estrategia de promoción de la salud y de prevención en las embarazadas sobre la importancia de los controles prenatales, la planificación familiar y el seguimiento del proveedor en la salud de la embarazada.

\section{Conclusiones}

En el espacio de formación de la Especialidad en Gestión de Servicios de Salud y Seguridad Social se pretende que los participantes aprendan a detectar, analizar, sistematizar y dar respuestas; para luego ser capaces de desarrollar proyectos de intervención que sirvan como solución a una situación problemática dentro del sector salud donde se desenvuelven profesionalmente. De esta manera no se limita a los participantes a identificar el problema y sus causas, sino que se propició llevarlos a que estén en condiciones para dar respuestas efectivas a esta situación y a otras similares. 
La naturaleza social del proyecto de formación guarda relación con el hecho de que el Área de Ciencias de la Salud del INTEC (a través del Programa Intec de Salud y Seguridad Social) tiene un interés especial en evaluar las situaciones gerenciales y problemas del área materno-infantil. Por tanto se harán intervenciones en las realidades que fueron detectadas; como por ejemplo, la alta incidencia de la mortalidad materna e infantil, o el mejoramiento de la calidad de vida durante el embarazo, el puerperio y los primeros días de vida del infante, cuya solución aún requiere de grandes esfuerzos intersectoriales y de la participación social. Se procura seguir formando gestores con competencias, que sean sensibles y con el compromiso para impulsar los cambios que requieren el sistema Nacional de Salud y el Sistema Dominicano de la Seguridad Social.

Esta experiencia de construcción colectiva estuvo dirigida por un equipo de una academia y por un equipo docente que se comprometió con el proceso de trabajo para la formación de gestores con capacidad y poder lograr la transformación de los sistemas. Todos están empoderados del macro-objetivo común y que nos conduce a intercambiar conocimientos y experiencias: Garantizar el derecho a la salud de la población, la acción intersectorial y la participación social en salud. Al principio se presentó como una utopía (lo era y lo sigue siendo, según Galeano):

\section{La utopía...}

Ella está en el horizonte, me acerco diezpasos y ella se aleja diezpasos más, y por más que yo me acerque nunca la alcanzaré. ...¿Para qué sirve la utopía? Para eso sirve, para caminar... 


\section{Referencias bibliográficas}

Díaz Bordenave, J. (2007). Algunos factores pedagógicos. Sistematización del Taller de reflexión crítica sobre la práctica pedagógica para la adapatación del Curso Internacional para Gestores de Politicas de Recursos Humanos a la Realidad de los Paises Andinos (pp.16-21). Lima: Universidad Nacional Mayor de San Marcos.

Instituto Tecnológico de Santo Domingo. (s.f.). Documento de orientación para el proceso de Trabajo de Campo Supervisado. Post-grado gestion de servicios de Salud y Seguridad Social. Santo Domingo: Instituto Tecnológico de Santo Domingo.

Navas, N. (1995). Metodología de la problematización: Una alternativa metodológica apropiada para la enseñanza superior. Semina: CL1. Soc./Hum., 16, 9-19. 


\section{Maritza Altagracia Martínez Rodríguez}

Dra. en Medicina (1985) egresada de la Universidad Autónoma de Santo Domingo (UASD). Tiene una especialidad en Educación Sexual (1986) por INSAPEC; maestría en Salud Pública por la UASD y especialidad en Gerencia Social de Servicios de Salud y Seguridad Social; es profesora de la carrera de Medicina y de post-grado en el INTEC y profesora adjunta de la Escuela de Salud Pública de Salud; maestría de Salud Pública y la especialidad de Salud Ocupacional en la UASD. En la actualidad coordina el Programa INTEC de Salud y Seguridad Social (PROISS) y la Especialidad en Gestión de Servicios de Salud y Seguridad Social. Con experiencia de trabajo con el Ministerio de Salud y consultorías realizadas con ONG, organismos y agencias de cooperación (OPS/OMS, USAID, Banco Mundial, BID, FNUAP, UNICEF, Cooperación Española) e instituciones académicas.

Email: maritzam1026@yahoo.com 
